\title{
Implementation and evaluation of a multifunctional telemedicine system in NTUH
}

\author{
Chung-Chih Lin a, Heng-Shuen Chen ${ }^{a}$, Ching-Yu Chen ${ }^{\text {a }}$, Sheng-Mou Hou ${ }^{\mathrm{b}, *}$ \\ a Department of Medical Informatics, Collage of Medicine, National Taiwan University, Taipei, Taiwan \\ ${ }^{\mathrm{b}}$ Department of Orthopedic Surgery, School of Medicine, National Taiwan University, No 1, Jen Ai Road, Sec. 1, \\ Taipei 100, Taiwan
}

\begin{abstract}
In this article, we proposed a multifunctional telemedicine system supporting both telediagnosis and teleconsultation services. We attempted not only to insure that the implementation of this system satisfied most requirements, but also to evaluate the impact of the system. With regard to system architecture, we designed a unified multimedia database to store all types of data and used two kinds of network (ATM and ISDN) for different possible applications. As for data transmission, the REFRESH and PREFETCH mechanisms were implemented to enhance data transfer efficiency. A total of 1107 consultations employing the telemedicine system were performed during the past 3 years. This technology was used most frequently for radiology consultation $(32.7 \%, n=362)$ and ultrasonic examination $(19.5 \%, n=216)$. An evaluation of the impact on diagnosis (507 valid cases) indicated that the diagnosis in 80 cases $(15.78 \%)$ were altered on the basis of second opinions from a medical center; and the number of patients transferred to the medical center was reduced from $24(4.7 \%)$ to eight cases. Most of the rural-site physicians $(97 \%)$ thought that they did benefit from specialists' experience and knowledge via the telemedicine system. Based on 431 valid questionnaires, the number of the patients with confidence in the telemedicine system at their local healthcare center increased from $72.6 \%$ to $87.5 \%$. Overall, more than $90 \%$ of patients and physicians believed that the system was valuable and provided satisfactory services. (C) 2001 Elsevier Science Ireland Ltd. All rights reserved.
\end{abstract}

Keywords: Telemedicine; ATM; ISDN

\section{Introduction}

Telemedicine can be defined as a system that provides medical services over long distances. Depending on the mode of operation,

\footnotetext{
* Corresponding author. Tel.: + 886-2-2397088-2190; fax: $+886-2-23971021$.

E-mail address: shengmou@ha.mc.ntu.edu.tw (S.-M. Hou).
}

telemedicine can support two types of healthcare services. One is off-line telediagnosis and the other is real-time teleconsultation. Telediagnosis can be performed asynchronously. That is, the specialist can make the diagnosis at his convenience and then forward the results to a physician at a remote site. Highquality medical media presentation is the most important issue in this mode. During a 
teleconsultation, the physicians at a remote site and the specialist in a medical center discuss the patient's records interactively. A real-time videoconferencing system and a document-sharing mechanism are the most essential elements of the teleconsultation mode [1].

The most frequent clinical application of telemedicine is in radiology. Telediagnostic services in radiology are now termed teleradiology. Teleradiology has been practiced in the Nordic countries since 1991 [2,3]. Medical information and images formerly were transmitted through a 64-kbps telephone line from a rural health center to a medical center. Thanks to recent improvements in network technology, a large amount of medical information can currently be transferred back and forth at a higher transmission rate [4-6]. Apart from teleradiology, clinical applications of telemedicine also include telecardiology, telepathology, teleneurology and telepsychiatry $[1,7]$. In these teleconsultation modes, real-time discussion between physicians in remote areas and specialists at a medical center is a very important aspect. Thus, the system must be capable of transferring audio and video signals, as well as diagnostic images and bio-signals, both simultaneously and synchronously.

The National Taiwan University Hospital (NTUH) has been developing a telemedicine system since 1995 for the purpose of improving healthcare in rural areas [8]. Compared with the systems described in previous studies, the NTUH telemedicine system is constructed to serve multiple functions and to support both telediagnosis and teleconsultation. The departments involved include radiology, internal medicine, surgery, dermatology, family medicine, gynecology and psychiatry. Our project attempts not only to insure that this system satisfies most requirements in the many applications, but also to evaluate the influence and impact of telemedicine. This paper is a retrospective evaluation of the performance of this system in terms of both patient's and doctor's satisfaction, stability of system and clinic benefits.

\section{Methods and materials}

\subsection{The Infrastructure of the NTUH telemedicine project}

Hampered by mountain and island geography, medical resources are poorly distributed in rural areas of Taiwan. Some physicians are reluctant to serve in rural areas because of the feeling of isolation and the lack of opportunities for continuing medical education (CME). People living in rural areas thus often receive relatively inadequate healthcare. We have therefore built the NTUH telemedicine system in order to alleviate these problems. Its specific goals are: (1) to improve medical service in rural areas; (2) to reduce the frequency of referrals; (3) to provide CME opportunities to the physicians in rural areas; and (4) to share advanced knowledge and expertise from the medical center. Using this channel of communication, physicians at a rural site can receive diagnostic opinions through telemedicine and obtain advanced medical knowledge from specialists at the medical center. Better healthcare and reduced transportation costs for patients are also expected benefits.

The architecture of the NTUH telemedicine system is shown in Fig. 1. The system includes remote sites (e.g. Chinshan Health Station and National Chung Kung University Hospital), an information center and teleconsultation/telediagnosis rooms. At a remote site, the data acquisition and teleconferencing systems are the most important means of providing patients' electronic infor- 
mation to the referred medical center. The NTUH information center is equipped with a multimedia database management system responsible for maintenance and backup of medical information from rural healthcare centers. The teleconsultation rooms (I), equipped with a dual-monitor viewing station and a teleconferencing system, supports the transmission of medical data from Chinshan Health Station. The main task of the teleradiology room (II) is to make asynchronous telediagnosis. Thus, only a dual-monitor viewing station is needed here, not a teleconferencing system. Since the teleconsultation room (III) must provide multi-point communications for case discussions, networking is the primary concern. In addition, the viewing station is equipped with only a standard PC with a single monitor.

\subsection{Image acquisition}

Digitization of medical images obtained via various modalities is the most complicated aspect of preparing multimedia medical information. Depending on the presentation method, medical images can be divided into two categories. One category consists of still images (e.g. CT, MR, and X-ray), and the other consists of dynamic images (e.g. ultrasound, cardioangiography, and endoscope) [9-12]. In order to standardize still images,

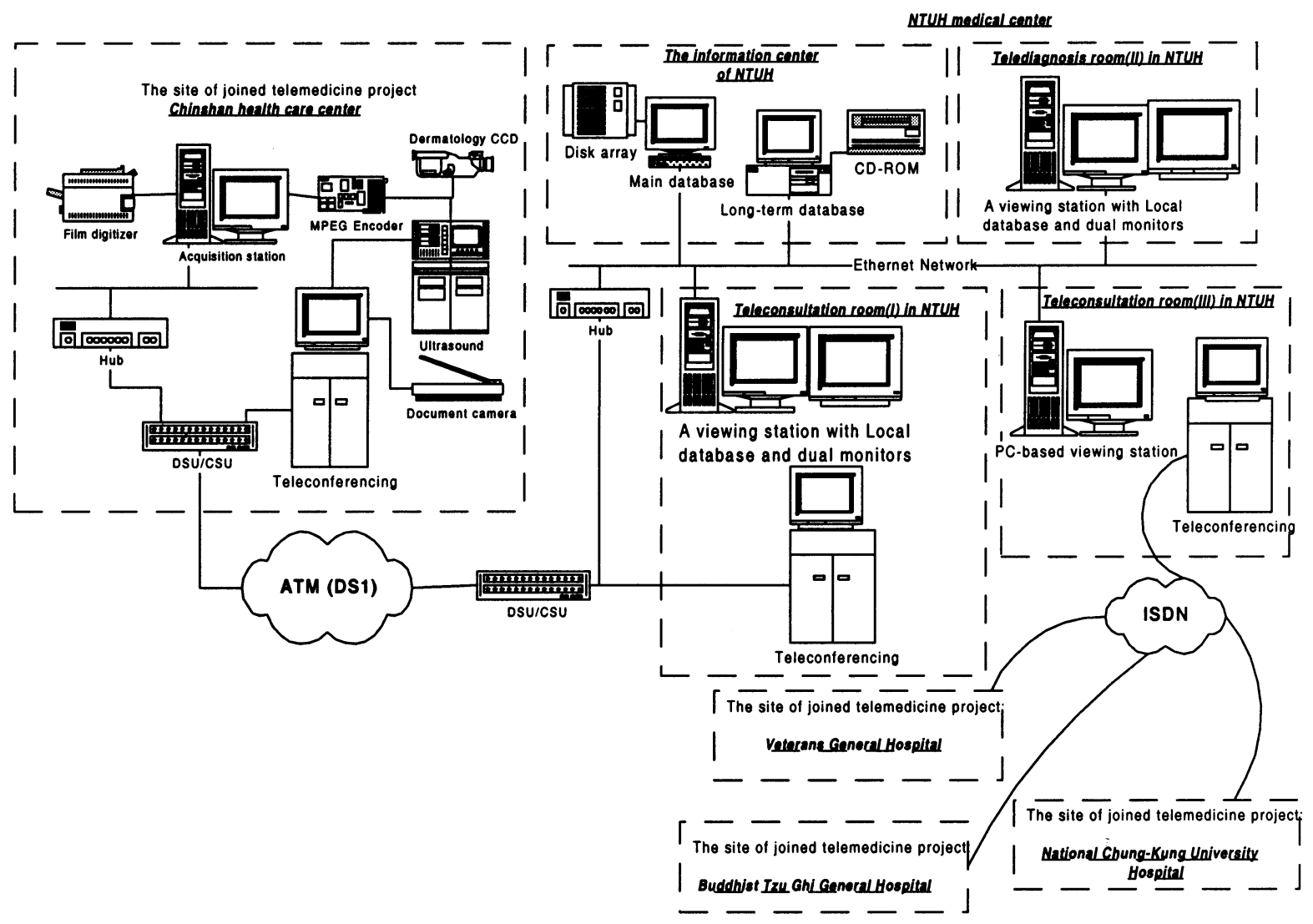

Fig. 1. The architecture of the NTUH telemedicine system. 


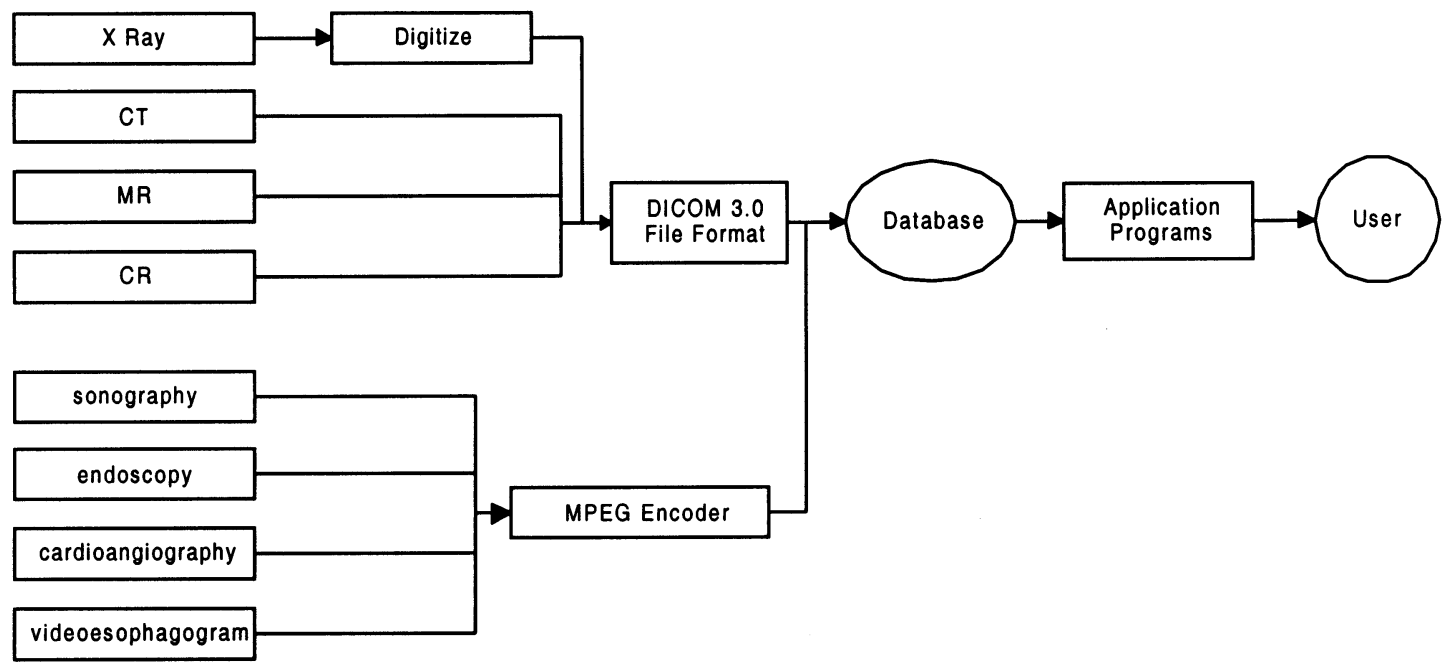

Fig. 2. The workflow of image acquisition.

all images are converted to the DICOM 3.0 format before storage (shown in Fig. 2), which, thanks to homogeneous display type, can greatly reduce system development effort [13]. Although the standardization of dynamic images was not discussed in previous studies as much as that of still images, it is at least equally important in such clinical practices such as cardiology. In the previous studies [14], the dynamic images were reported as recorded on tape or digitized with an image grabber on a frame-by-frame basis. Lack of unified management could make it difficult to share images simultaneously. In our system, dynamic images are digitized with an MPEG encoder and stored in multimedia databases with data of other types.

\subsection{Unified database and patient information package}

The database must meet five requirements to satisfy telemedicine standards. First, the most concise data fields are used to keep patients' medical records on track. This data should support both the patient-oriented and clinical problem-oriented query strategies. Secondly, the database should be designed for multiple applications. The data fields must be easily adaptable to fit a variety of requirements for different departments or units. Thirdly, the database information (i.e. text, images, and video data fields) should be easily manipulated and accessible with high efficiency. Fourthly, the database should provide a uniform programming interface to reduce development and maintenance effort. Finally, the database should be powerful enough to permit multiple users to access multimedia data concurrently.

In order to manage and search patients' information more efficiently, we define a $\mathrm{Pa}$ tient Information Package (PIP) as a concise data set that records a patient's medical information for each visit [15]. A PIP data set is represented by the tuple $(I, N, P, S, L)$ where $I$ is for PIP identification; $N$ is for the patient's chart number of the order form; $P$ is a specific number related to the clinical problem; $S$ is for tracing the effect of therapies; 
and $L$ is for a link to the previous PIP. From the point of implementation, each PIP relates to a whole set of subjective and objective, assessment and planning objects created according to specific clinical diagnosis or therapy (an ER diagram is shown in Fig. 3). Once a medical service (i.e. radiological examination) is carried out, Subjective, Objective, Assessment and Plan (SOAP) procedures are performed and used to create the corresponding medical information [16]. This information is then filled into a specific PIP. A PIP is thereupon created and stored into the database with linkage to other PIPs by the unique numbering system. That is, a database designed for telemedicine can be constructed with a fully object-oriented structure. A PIP, on one hand, can be adapted to a variety of medical services or clinical exam- inations without any difficulty. On the other hand, it can be linked to other PIPs to integrate a specific patient's medical history or long-term case. Thus, a group of PIPs can be used to record the diagnostic and therapeutic history of a patient (patient-oriented) or to collect cases for a specific disease (problemoriented). This is how the database is able to satisfy the diversity and flexibility required of a telemedicine system.

Lastly, we use an Object-Relational Database Management System (ORDBMS) to manage all types of data. This system provides an extensible field capable of handling large objects directly through standard structural query language (SQL), which can not only simplify database system maintenance, but also improve consistency by providing a unified accessing mechanism for all types of data.

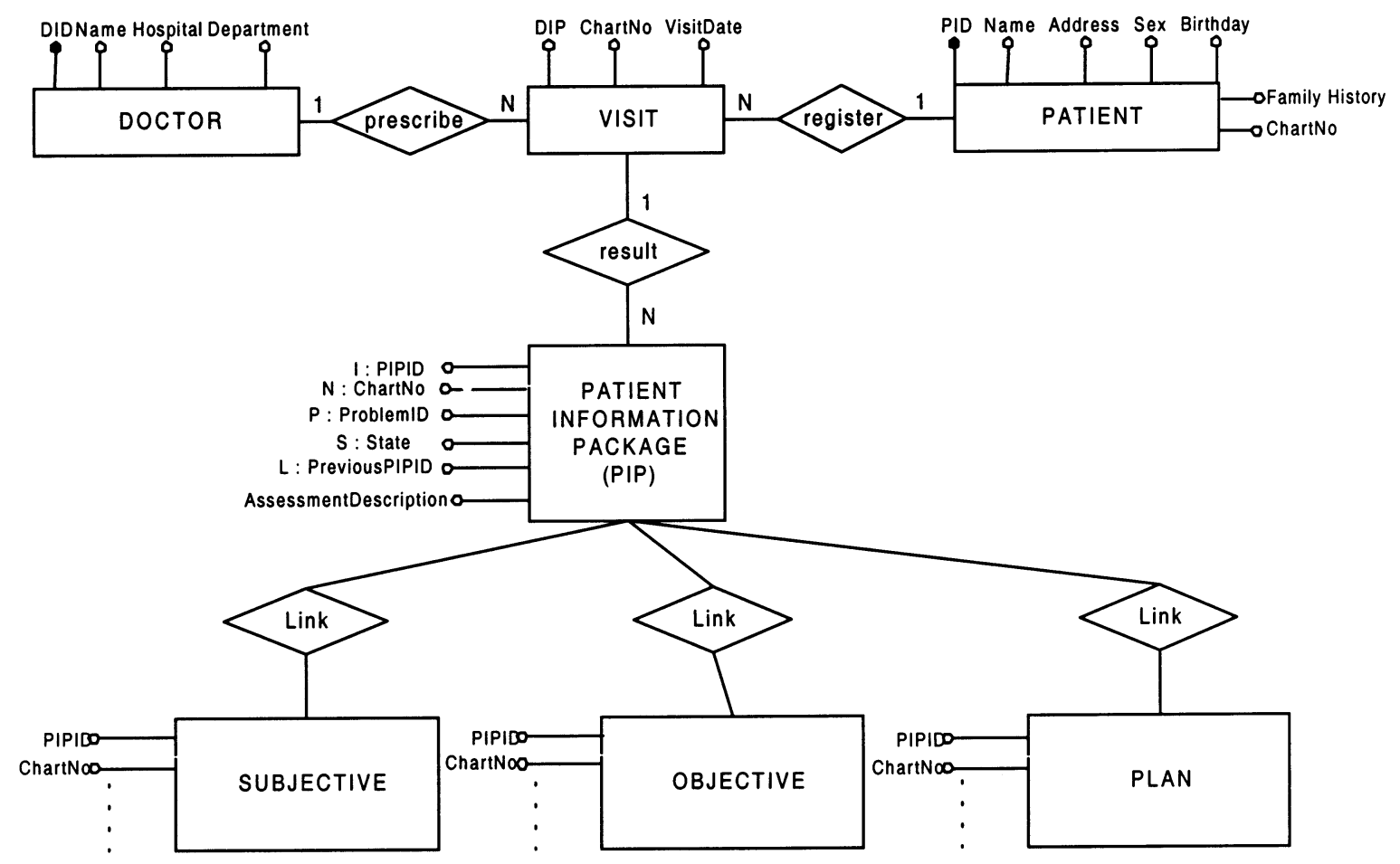

Fig. 3. Entity-relation (ER) diagram of PIP-based medical database 


\subsection{Networking}

To guarantee the bandwidth and service quality, telemedicine systems most frequency employ switch-based networking with a wide area network (WAN) architecture. Among a variety of switch-based networks, ATM and ISDN are the main choices for telemedicine. ATM networking provides bandwidth with a range from 1.54 Mbps (DS1) to $2.4 \mathrm{Gbps}$ (OC-48), which is wide enough to transfer most patient information real-time through one channel, but a relatively high expense is inevitable. On the other hand, ISDN provides bandwidth with a range from $64 \mathrm{Kbps}$ (BRI) to 1.54 Mbps (PRI). The advantages of ISDN are it's low cost and more hardware and software support. However, ISDN does provide less bandwidth than ATM. Therefore, the optimal networking configuration for telemedicine must be determined according to the specific situation, and should attempt to provide maximal communication flexibility. For communicating with a fixed site or providing teleradiology services in asynchronous mode (i.e. Chinshan Health Station as shown in Fig. 1), an ATM-T1 leased line connection is used to provide higher bandwidth for image data transmission. For communicating with a mobile site or providing multi-point teleconferencing (i.e. National Chung Kung University Hospital as shown in Fig. 1), ISDN networking is used when necessary.

\subsection{Data transmission}

It takes more than 10 Mbytes memory and approximately $100 \mathrm{~s}$ to transfer an uncompressed X-ray film image via a T1 channel [6]. Thus, how to provide high-efficiency image transmission is an important issue in implementation of telemedicine systems. To insure a high level of efficiency, both the rural oper- ating console and the viewing station at the NTUH medical center have set up a local database, and also bear responsibility for REFRESH and PREFETCH (Fig. 4a) tasks. REFRESH is a data forwarding mechanism that validates and stores a patient's data records in the local database of the operating console when a patient is registering for a new visit (shown in Fig. 4b). When patient data records are transferred through the WAN, REFRESH will book the data fields that are successfully transferred to and stored in the main database in the medical center. Once the network has been disconnected, not all data records must be sent later, only those records that have failed to be transferred according to the look-up-table of REFRESH. This can save a substantial amount of data transmission time. For presenting a patient's data to specialists as soon as possible, a PREFETCH mechanism is implemented in the viewing station at the medical center. For teleconsultation, the PREFETCH mechanism precedes the consultation. It accesses the medical records according to a schedule. In telediagnosis, multimedia medical records may also be prefetched when the diagnostic report has not yet been completed. This allows the presented data records be accessed directly from the local database at the viewing station, and certainly improve the efficiency of data processing and representation.

\subsection{Data presentation}

Acceptance of a system depends mainly on the quality of the interface to the users $[16,17]$. In the design of telemedicine systems, user interfaces should achieve high-quality data presentation and transmission. To satisfy the requirement of image display for clinical diagnosis, a dual-monitor viewing station is installed. One monitor has high-reso- 


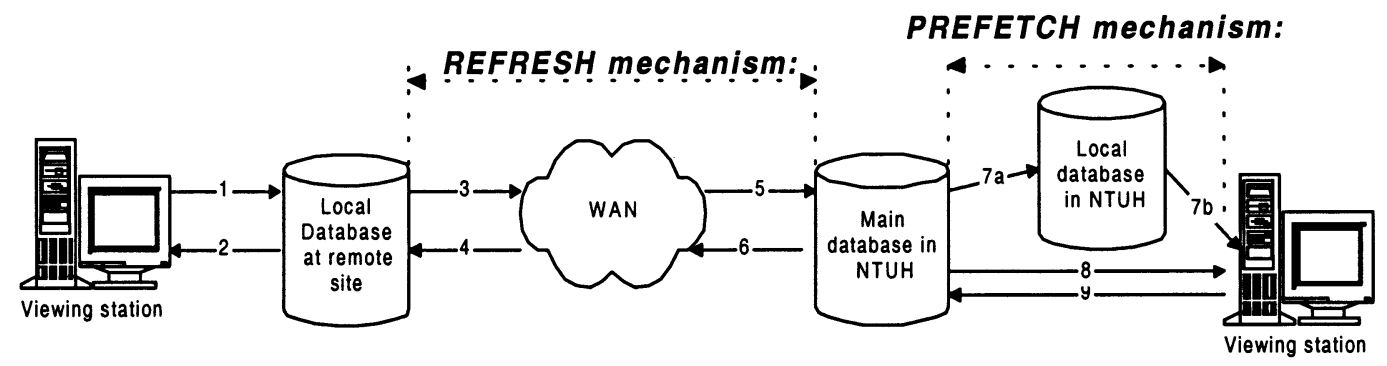

(a)

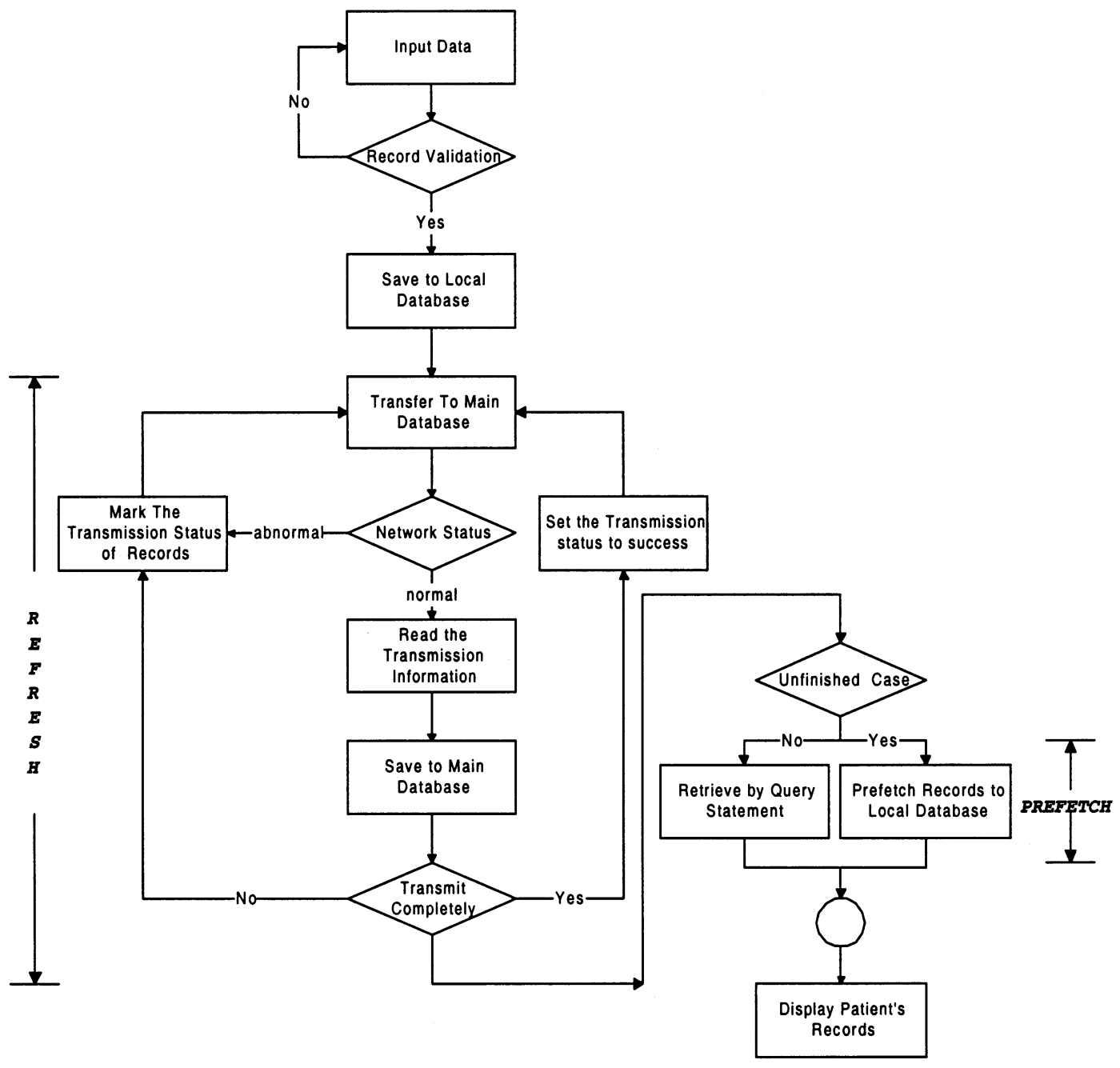

(b)

Fig. 4. (a) The diagram of REFRESH and PREFETCH mechanism. (b) The flowchart of data transmission. 


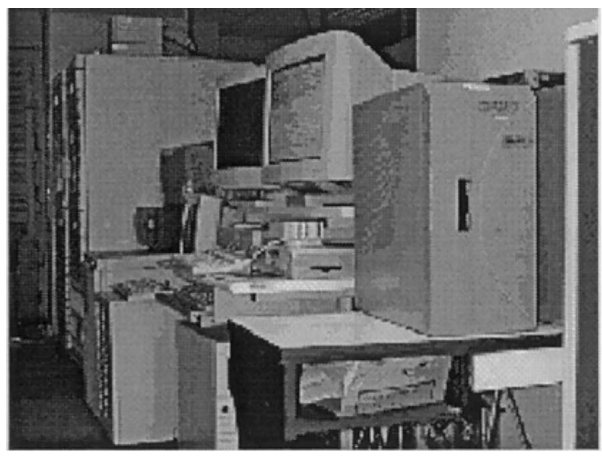

(a)

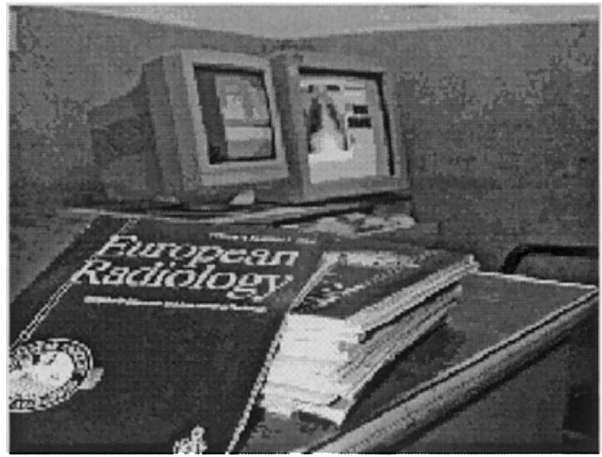

(c)

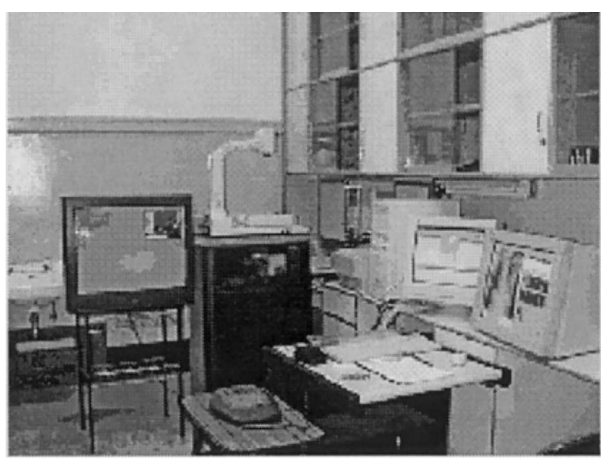

(b)

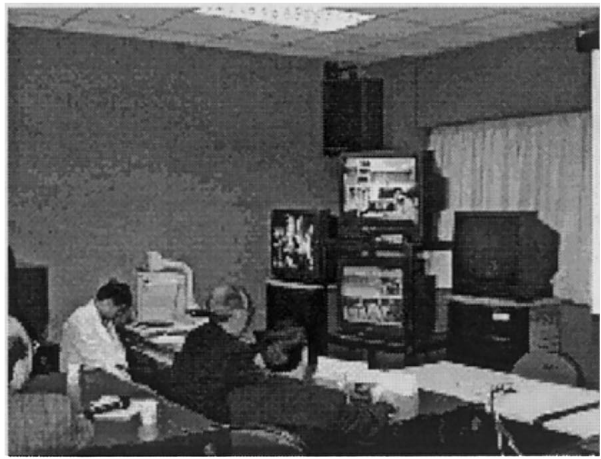

(d)

Fig. 5. (a) The information center of NTUH in Fig. 1. (b) The Teleconsultation room (I) is equipped with dual-monitor viewing station and an ATM-DS1 broadband communication channel. (c)The telediagnosis room (II) is equipped with only a dual-monitor viewing station. (d) The teleconsultation room (III) is equipped with a PC and it can support multi-point teleconferencing based on ISDN networking.

lution and high-illumination features to facilitate the display of medical images. The maximal spatial resolution of the displayed image exceeds $2048 \times 2487$ and 12 bits per pixel. The other monitor is an SVGA monitor used to display the MPEG video and text data. The two-monitor viewing station can now support the display of dynamic images encoded in MPEG format, still images such as $\mathrm{X}$-ray, CT, and MR images, and text editing fields. In addition to image display, the telemedicine system must provide basic tools for image manipulation. For example, zooming image in and out, flipping image verti- cally and horizontally, rotating image 90 , 180 , or $270^{\circ}$, and adjusting window and level of images are all essential features.

\section{Results}

The NTUH telemedicine system (shown in Fig. 5) started its operation since February 1996 [18-21]. The system worked normally and smoothly in most of cases $(97.2 \% n=$ 688, 708 valid cases). The system downtime of $2.5 \%(n=18)$ cases were less than $5 \mathrm{~min}$. There were only two cases when data and 
video signal transmission was lost for more than $10 \mathrm{~min}$, and both cases occurred during the first year of implementation. At that time, the main reason for signal loss was unstable channels for broadband WAN networking. To evaluate the quality of dynamic ultrasonic images transmitted through the communication channels, the video signals at both sites (Chinshan Health Station and NTUH Medical Center) were recorded in real-time using high quality SVHS video tape recorders [22]. The two tapes were played off-line at the same time. The quality of the video signals was then evaluated subjectively by a specialist. Based on 34 studies, although the video quality was degraded by the CODEC during transmission at the NTUH site, this did not affect the diagnostic accuracy. The result showed that ultrasonic diagnosis through telemedicine system is feasible. The only pitfall was that the examination time was longer than in conventional examinations.

Table 1 shows the gender, age and department of patients included in the telemedicine study during the past 3 years. The total of 1107 cases consisted of $46.98 \%$ female and $53.02 \%$ male cases. $33.6 \%$ of the patients were more than 65 years old, showing that seniors benefited the most from telemedicine. Table 1 also shows that the most frequent applications of telemedicine were for teleradiology $(32.7 \%, n=362)$ and ultrasonic examination $(19.5 \%, n=216)$.

Based on the results of 431 valid questionnaires from the patients (results shown in Table 2), more than $87 \%$ of the patients

Table 1

The statistics of patients' gender, age and visit department in three years

\begin{tabular}{|c|c|c|c|c|}
\hline & $\begin{array}{l}\text { First year } \\
\text { Feb.-Dec } 1996 \\
\text { Number }(\%)\end{array}$ & $\begin{array}{l}\text { Second year } \\
\text { Jan. 1997-Dec. } 1998 \\
\text { Number }(\%)\end{array}$ & $\begin{array}{l}\text { Third year } \\
\text { Jan.--July } 1998 \\
\text { Number }(\%)\end{array}$ & $\begin{array}{l}\text { Total } \\
\text { Number }(\%)\end{array}$ \\
\hline \multicolumn{5}{|l|}{ Gender } \\
\hline Male & $266(42.83 \%)$ & $159(49.7 \%)$ & $94(50.8 \%)$ & $519(46.98 \%)$ \\
\hline Female & $355(57.17 \%)$ & $161(50.3 \%)$ & $91(49.2 \%)$ & $587(53.02 \%)$ \\
\hline \multicolumn{5}{|l|}{ Age (years) } \\
\hline $1-17$ & $49(8.5 \%)$ & $33(10.5 \%)$ & $10(5.4 \%)$ & $92(8.5 \%)$ \\
\hline $18-24$ & $28(4.8 \%)$ & $10(3.2 \%)$ & $6(3.2 \%)$ & $44(4.1 \%)$ \\
\hline $25-39$ & $100(17.3 \%)$ & $48(15.2 \%)$ & $22(11.9 \%)$ & $170(15.8 \%)$ \\
\hline $40-50$ & $67(11.6 \%)$ & $46(14.6 \%)$ & $26(14.1 \%)$ & $139(12.88 \%)$ \\
\hline $51-64$ & $144(24.9 \%)$ & $76(24.1 \%)$ & $51(27.6 \%)$ & $271(25.12 \%)$ \\
\hline $65+$ & $191(33 \%)$ & $102(32.4 \%)$ & $70(37.8 \%)$ & $363(33.6 \%)$ \\
\hline \multicolumn{5}{|l|}{ Department } \\
\hline X-Ray & $189(31.4 \%)$ & $123(38.4 \%)$ & $50(27 \%)$ & $362(32.7 \%)$ \\
\hline Ultrasound & $110(18.3 \%)$ & $59(18.4 \%)$ & $47(25.4 \%)$ & $216(19.5 \%)$ \\
\hline Internal medicine & 0 & $2(0.6 \%)$ & $19(10.3 \%)$ & $21(1.9 \%)$ \\
\hline Surgery & $7(1.2 \%)$ & $9(2.8 \%)$ & $1(0.5 \%)$ & $17(1.5 \%)$ \\
\hline Gynecology & $3(0.5 \%)$ & $24(7.5 \%)$ & $3(1.6 \%)$ & $30(2.7 \%)$ \\
\hline Family medicine & $254(42.2 \%)$ & 0 & 0 & $254(22.9 \%)$ \\
\hline Dermatology & $17(2.8 \%)$ & $21(6.6 \%)$ & $14(7.6 \%)$ & $52(4.7 \%)$ \\
\hline Rehabilitation & $8(1.3 \%)$ & $49(15.3 \%)$ & $46(24.9 \%)$ & $103(9.3 \%)$ \\
\hline Psychiatry & 0 & $16(5 \%)$ & $4(2.2 \%)$ & $20(1.8 \%)$ \\
\hline Teaching clinics & $14(2.3 \%)$ & $17(5.3 \%)$ & $1(0.5 \%)$ & $32(2.9 \%)$ \\
\hline
\end{tabular}


Table 2

Results of patient questionnaire on telemedicine system ${ }^{\mathrm{a}}$

\begin{tabular}{|c|c|c|c|c|c|c|c|}
\hline & Very much & Yes & $\begin{array}{l}\text { Neutral or } \\
\text { comment no }\end{array}$ & No & Totally not & Positive rate & $\begin{array}{l}\text { Effect of } \\
\text { acquaintance } \\
\text { ( } Z \text { value of } \\
\text { Mann-Whitney) }\end{array}$ \\
\hline Q1: Accept the service of telemedicine? & $26.9 \%$ & $60.6 \%$ & $9.0 \%$ & $3.0 \%$ & $0.5 \%$ & $87.5 \%$ & $-2.2470^{*}$ \\
\hline Q2: Can 'see' the specialist clearly? & $50.6 \%$ & $44.8 \%$ & $0.9 \%$ & $3.2 \%$ & $0.5 \%$ & $95.4 \%$ & $-1.0817-$ \\
\hline Q3: Can 'hear' specialist's voice clearly? & $61.4 \%$ & $34.1 \%$ & $0.5 \%$ & $3.2 \%$ & $0.5 \%$ & $95.5 \%$ & $-0.8143-$ \\
\hline Q4: Feel easy to talk to the specialist? & $38.1 \%$ & $52.0 \%$ & $3.9 \%$ & $6.0 \%$ & $0.0 \%$ & $90.1 \%$ & $-2.1269^{*}$ \\
\hline Q5: Communicate with specialist well? & $36.0 \%$ & $52.9 \%$ & $5.3 \%$ & $5.3 \%$ & $0.5 \%$ & $88.9 \%$ & $-2.3084^{*}$ \\
\hline Q6: Believe the diagnosis result? & $26.2 \%$ & $66.6 \%$ & $6.0 \%$ & $1.2 \%$ & $0.0 \%$ & $92.8 \%$ & $-2.7894 * *$ \\
\hline Q7: Will visit again 'without' specialist? & $10.0 \%$ & $62.6 \%$ & $11.1 \%$ & $15.3 \%$ & $0.9 \%$ & $72.6 \%$ & $-1.2183-$ \\
\hline Q8: Will visit again 'with' specialist? & $32.7 \%$ & $54.8 \%$ & $8.8 \%$ & $3.2 \%$ & $0.5 \%$ & $87.5 \%$ & -0.7064 \\
\hline Q9: Satisfy the service of telemedicine? & $30.2 \%$ & $63.8 \%$ & $4.4 \%$ & $1.6 \%$ & $0.0 \%$ & $94.0 \%$ & $-2.0912 *$ \\
\hline
\end{tabular}


accepted medical service through the telemedicine system. More than $95 \%$ of them stated that they could 'see' or 'hear' the doctor in the monitor clearly. Ninety percent of the patients said they were not afraid to describe their problems to the specialist in the monitor. The number of patients who were willing to receive care in the local healthcare center increased from $72.6 \%(\mathrm{Q} 7)$ to $87.5 \%(\mathrm{Q} 8)$. Ninety-four percent of the patients expressed overall satisfaction with the telemedicine system. This showed that most patients accepted such a diagnostic modality. In the questionnaire, we also evaluated whether the results were altered by the patient's acquaintance with the referred specialist in the medical center (shown in the last column of Table 2). The results showed that patients with less acquaintance with the referred specialist felt more hesitant to describe their illness and had difficulty in communicating with the referred specialist (Q4 and Q5). On the other hand, patients who were acquainted with the referred doctor had more confidence in the diagnosis result, as displayed in Q1, Q6 and Q9 of Table 2. Thus, telemedicine is more suitable for the long-term follow-ups and for chronic patients in special clinics.

Based on 507 valid returned questionnaires, most rural-site physicians $(95.3 \%$, $n=483$ ) needed specialists' advice to help with the diagnosis. The remaining $4.7 \%$ $(n=24)$ wished to transfer their patients to NTUH for further diagnosis or treatment. As for the results of diagnosis, $15.78 \%(n=$ 80 ) of the cases were altered in accordance with advice from the medical center. The number of patients transferred to the medical center was reduced from 24 to 8 cases. The remaining $83 \%(n=419)$ were either unchanged or left to await further investigation and examinations. Most of the ruralsite physicians $(97 \%)$ thought that they did benefit from advanced medical experience and knowledge from specialists available through the telemedicine system. These results showed that a telemedicine system could help rural doctors by providing suggestions and diagnostic advice from experts. The system also provided additional information for the rural-site physicians in order to help them decide if their patients needed to be transferred. A high percentage $(98.4 \%$, $n=501)$ of the rural-site physicians were satisfied with the judgements concerning transferring their patients derived through the telemedicine system. The above results showed the feasibility of telemedicine systems to improve the quality of medical services in rural areas.

Based on the 705 valid questionnaires on teleconsultation from the referred specialists at the medical center, $92.8 \%$ felt satisfied with their communication with doctors at rural sites. The error rate caused by misunderstandings during this communication was only $0.9 \%(n=6)$, which shows that doctors at both sites could communicate effectively through the system. Finally, general satisfaction with the results of telemedicine system was assessed. Most $(91.6 \%, n=644)$ of the specialists were satisfied with the results of telemedicine. According to these findings, dissatisfaction occurred mostly in cases of child psychiatry and dermatology, when specialists were not satisfied with quality of the CCD camera. As a means of solving this problem, child psychiatry specialists suggested adding more cameras during teleconsultation [23]. This could expand the field of view as wide as possible, and cover all the areas the children could reach. In the case of dermatology diagnosis, the doctors proposed using high-quality wireless cameras to improve image quality at any given position and angle. 


\section{Conclusion}

The NTUH telemedicine system can not only provide teleconsultation services for multiple departments, but also fulfil the requirements of second-generation teleradiology, since it is capable of DICOM services, image displaying and manipulation, and database management for patients' medical records [24]. The number of telemedicine cases has exceeded 1100 during the last 3 years. More than $90 \%$ of patients and doctors felt that the system was valuable and provided satisfactory services. In the future, the system will continue to provide extended medical services to rural areas. Furthermore, an effort will be made to continue the development of web-based telemedicine so as to extend the system to an even broader range of applications.

\section{Acknowledgements}

This research was supported by the grant "an NII pilot study of telemedicine" from the Department of Health, Executive Yuan, Taiwan. We would like to thank Dr. YeunChung Chang, Dr. Yi-Lian Lui, and Dr. Fer-Ran Gou for their enthusiastic involvement in this research.

\section{References}

[1] E.C. James, Y. Kim, Multimedia systems for telemedicine and their communications requirements, IEEE Commun. Mag. 1 (1996) 20-27.

[2] T. Paakkala, J. Aalto, V. Kähärä, S. Seppänen, Diagnostic performance of a teleradiology system in primary health care, Comput. Methods Programs Biomed. 36 (1991) 157-160.

[3] J. Viitanen, T. Sund, E. Rinde, J. Stoermer, M. Kormano, J. Heinila, et al., Nordic teleradiology development, Comput. Methods Programs Biomed. 37 (1992) 273-277.
[4] H. Handels, C. Busch, J.C. Hahn, V. Kuhn, J. Miehe, S.I. Pöppl, et al., KAMEDIN: a telemedicine system for computer supported cooperative work and remote image analysis in radiology, Comput. Methods Programs Biomed. 52 (1997) 175-183.

[5] S.J. Dwyer, A.W. Templeton, Teleradiology using switched dialup networks, IEEE J. Select. Areas Commun. 10 (1992) 1161-1172.

[6] H.K. Huang, Teleradiology technologies and some service models, Comput. Med. Imag. Graph. 20 (1996) 59-68.

[7] B.H. Guze, R. Estep, C. Fisher, Telemedicine: a review of its use and a proposal for application in psychiatric consultation, Med. Inform. 20 (1995) $1-18$.

[8] C.T. Liu, C.C. Lin, J.M. Wong, S.K. Chiou, R.S. Chen, J.H. Chen, et al., Design and evaluation of a telediagnosis system, Biomed. Eng. Appl. Basis Commun. 9 (1997) 52-60.

[9] T.C. Wong, H.K. Huang, Networked multimedia for medical imaging, IEEE Multimedia 4 (1997) $24-35$.

[10] H.K. Huang, R.L. Arenson, S.L. Lou, W.K. Wong, K.P. Andriole, T.M. Bazzill, et al., Multimedia in the radiology environment: current concept, Computerized Med. Imaging Graphics 18 (1994) 1-10.

[11] G.F. Egan, Z.Q. Liu, Computers and networks in medical and healthcare systems, Comput. Biol. Med. 25 (1995) 355-365.

[12] T. Kitanosono, Y. Kurashita, M. Honda, T. Hishida, H. Konishi, M. Mizuno, et al., The use of multimedia in patient care, Comput. Methods Prog. Biomed. 37 (1992) 259-263.

[13] Digital Imaging and Communications in Medicine (DICOM) Version 3.0 American College of Radiologists/National Electrical Manufacturers Association, 1993.

[14] K. Kurisu, Y. Hishikawa, M. Izumi, M. Taniguchi, N. Kamikonya, N. Nakao, Personal computer-based small PACS for radiotherapy analog image filing system for radiotherapy, Comput. Methods Prog. Biomed. 43 (1994) 71-73.

[15] C.C. Lin, J.R. Duann, C.T. Liu, H.S. Chen, J.L. $\mathrm{Su}$, J.H. Chen, A unified multimedia database system to support telemedicine, IEEE Trans. Inform. Technol. Biomed. 2 (1998) 183-192.

[16] R.E. Rakel, Textbook of Family Practice, 5th ed., Philadelphia Saunders, 1995.

[17] H.U. Lemke, G. Faulkner, M. Krauss, Development towards multimedia medical workstations, Comput. Med. Imag. Graph. 18 (1994) 67-71. 
[18] Y.C. Chang, Y.L. Liu, H.M. Liu, C.Y. Chen, R.S. Chen, S.M. Hou, Interactive tele-consultation in abdominal sonography: preliminary report based on 147 cases, Formosan J. Med. 2 (1998) 397-402.

[19] H.S. Chen, F.R. Guo, C.T. Liu et al., Telemedicine Project in Taiwan: Development and Prospect. Health Congress Asia'98, 1998 Singapore. Singapore: AIC Worldwide, 1998, pp. 1-9.

[20] H.S. Chen, Information Systems in Primary Care in Taiwan [Abstract].WONCA Asia Pacific Regional Conference, 1999, Taipei. Taipei: ChineseTaipei Association of Family Medicine, 1999, p. 102.

[21] H.S. Chen, F.R. Guo, J.H. Chen et al., Progress and Prospect of Telemedicine in Taiwan [Abstract]. WONCA Asia Pacific Regional Conference, 1999,
Taipei. Taipei: Chinese Taipei Association of Family Medicine, 1999, p. 104.

[22] Y.C. Chang, H.S. Chen, C.C. Lin et al., Current Development in Teleradiology and Teleconsultation of the NTU Telemedicine Network. 1999 NSC-NSF Joint Workshop on NGI Applications, Hsinchu. Hsinchu: National Center for High-Performance Computing, 1999, p. 31.

[23] W.T. Soong, An Evaluation of Telemedicine Consultation Service for Children with Autism, A Symposium in Honour of Professor Char-nie Chen, August 1998, Honk Kong.

[24] U.A. Schroter, U. Baur, O. Werner, M. Schwab, H. Muller, H.P. Meinzer, A three-generation model for teleradiology', IEEE Trans. Inform. Technol. Biomed. 2 (1998) 20-24. 